\title{
Evaluation of safety and efficacy of apatinib combination with chemotherapy for ovarian cancer treatment: a systematic review and meta-analysis
}

\author{
Jing $\mathrm{Qi}^{1 *}, \mathrm{E} \mathrm{Liu}^{1 /}$, Haiquan Yue ${ }^{1}$, Guang Chen ${ }^{2}$, Yuting Liu ${ }^{3}$, Jiahui Chen ${ }^{4 \wedge}$ \\ ${ }^{1}$ Department of Stomatology, Gansu Provincial People's Hospital, Lanzhou, China; ${ }^{2}$ Department of Stomatology, Affiliated Hospital of Northwest \\ University for Nationalities, Lanzhou, China; ${ }^{3}$ Department of Radiology, Gansu Provincial People's Hospital, Lanzhou, China; ${ }^{4}$ Department of \\ Clinical Skills Training Center, Clinical Academic Affairs Office, The First Hospital of Lanzhou University, Lanzhou, China \\ Contributions: (I) Conception and design: J Chen; (II) Administrative support: None; (III) Provision of study materials or patients: J Qi, E Liu; (IV) \\ Collection and assembly of data: J Qi, H Yue, G Chen; (V) Data analysis and interpretation: E Liu, G Chen, Y Liu, J Chen; (VI) Manuscript writing: \\ All authors; (VII) Final approval of manuscript: All authors. \\ "These authors contributed equally to this work. \\ Correspondence to: Jiahui Chen. Department of Clinical Skills Training Center, Clinical Academic Affairs Office, The First Hospital of Lanzhou \\ University, Lanzhou 730000, China. Email: cjh20201001@163.com.
}

\begin{abstract}
Background Apatinib in combination with chemotherapy (CT) has been used in the treatment of ovarian cancer (OC), however, the safety and efficacy are unclear. The study aims at systematic evaluation of the safety and efficacy of the apatinib targeted therapy in combination with CT for the treatment of patients with advanced OC.
\end{abstract}

Methods: Literature about randomized controlled clinical trials was searched using search engines such as PubMed, EMBASE, Web of Science, CNKI, the Cochrane Library, CBM, VIP and the Wanfang. We collected the related clinical studies of apatinib in combination with CT in the treatment of OC. The duration of the data retrieval related to clinical studies was from the database establishment to September 2020. Adverse reactions (ADRs) due to treatment, disease control rate (DCR), and that of objective response rate (ORR), were collected as indicators to show treatment outcomes. The literature was independently screened by two researchers. They extracted the data and evaluated the risk of biases of the included studies. Then, Revman 5.4 software was employed for performing the meta-analysis.

Results: Twelve randomized controlled clinical trials with 698 patients having an advanced stage of OC were included. The results revealed that in comparison with the treatment with only CT, apatinib targeted therapy combination with CT showed significant improvement in the patients' ORR [OR $=3.19,95 \%$ CI: (2.06, 4.94), $\mathrm{P}<0.00001]$ and DCR [OR =4.97, 95\% CI: $(2.90,8.52), \mathrm{P}<0.00001]$. The group that was treated with a combined therapy had shown proteinuria in higher amount $(\mathrm{OR}=3.08,95 \% \mathrm{CI}: 51.13-8.42$, $\mathrm{P}<0.00001)$, while the analyses of other ADRs, such nausea and vomiting (OR $=1.10,95 \% \mathrm{CI}: 0.67-1.79$, $\mathrm{P}=0.71)$, hand-foot syndrome $(\mathrm{OR}=1.73$, 95\% CI: 0.97-3.10, $\mathrm{P}=0.06)$, hypertension $(\mathrm{OR}=1.18,95 \%$ CI: 0.73-1.91, $\mathrm{P}=0.0 .51)$, diarrhea ( $\mathrm{OR}=1.05,95 \% \mathrm{CI}: 0.56-1.97, \mathrm{P}=0.87)$, leucopenia $(\mathrm{OR}=1.22,95 \%$ CI: $0.70-2.12, \mathrm{P}=0.48$ ), and myelosuppression ( $\mathrm{OR}=1.00,95 \% \mathrm{CI}: 0.28-3.62, \mathrm{P}=1.00$ ), did not show any significant difference $(\mathrm{P}>0.05)$.

Discussion: The effects of apatinib combination with CT for the treatment of OC are significantly better than the CT used alone in ORR and DCR, despite with a relative low incidence of adverse effects. However, due to the very low number of studies available, the results need to be further verified using a high-quality, large sample and long-term studies.

^ ORCID: 0000-0003-2010-6390. 
Keywords: Apatinib; chemotherapy (CT); combination; ovarian cancer (OC); meta-analysis

Submitted Jun 11, 2021. Accepted for publication Aug 19, 2021.

doi: 10.21037/apm-21-1662

View this article at: https://dx.doi.org/10.21037/apm-21-1662

\section{Introduction}

Ovarian cancer (OC) is a major reason for mortality in patients having gynecological malignant tumor (1), the mortality rate is second only to cervical cancer and uterine body cancer, ranking the third highest. Epidemiological survey shows that the annual incidence of new cases worldwide is 255 thousand, and the incidence rate is increasing by $0.1 \% /$ year in recent years (2). In 2018, more than 295,000 women worldwide developed epithelial ovarian cancer (EOC), of which more than 184,000 died (3). There is also a heterogenous group of rare ovarian tumours that affect mainly young patients-the non-EOCs. They are histologically and clinically distinct tumours, uncommon but not rare. Approximately $10 \%$ of the OC patients present with non-epithelial histology and those include germ cell tumours and sex-cord stromal tumours, each of which is subdivided into several histological subtypes (4). The treatment of OC includes surgery and postoperative platinum chemotherapy (CT) (5). Three or more cycles of neoadjuvant CT prior to debulking surgery and adjuvant $\mathrm{CT}$ is an alternative option for selected patients. Neoadjuvant CT offers the opportunity to test upfront chemosensitivity and to identify patients at higher risk of relapse. CT can effectively prevent the proliferation, invasion, and metastasis of tumor cells, but CT drugs can damage normal cells while killing tumor cells (6). This not only reduces the tolerance of patients to CT but also disturbs the survival time of patients and affects the quality of life (7). About $80 \%$ of OC patients will relapse and metastasize after standard treatment (8). The mean survival time was about 2 years (9). Therefore, there is an urgent need to explore new and effective treatment methods. The molecular advances have identified that defective DNA damage response is a defining hallmark of high grade EOC which led to specific interest in BRCA genes, which are involved in the repair of DNA double strand breaks via the homologous recombination (HR) repair pathway. Poly (ADP-ribose) polymerase (PARP) inhibition in BRCA mutant tumor cells could induce "synthetic lethality", based on the simultaneous targeting of two DNA repair pathways.
Pharmacologic PARP inhibition in HR deficient tumors exploits this vulnerability. Moreover, a separate spectrum of genetic abnormalities related to the HR pathway sensitizes BRCA wild-type cancers to PARP inhibition. This molecular basis includes mutations in certain genes beyond BRCA1/2, homozygous somatic loss, and whole genome properties such as genomic scarring. Olaparib, rucaparib, and niraparib have been already commercialized in different settings in OC. Veliparib does not yet have an approved label, and its use is being investigated mostly in combination with CT or targeted agents (10).

In recent years, small molecule targeted therapy has revealed broad prospects. Using as a small molecule, the apatinib targeted therapy can suppress the proliferation and migration process of endothelial cells, induced by a vascular endothelial growth factor (VEGF) via specific binding to a VEGF receptor 2 (VEGFR-2), and hence reduces the tumor microvessels' density (11). Some studies have confirmed that it can't only be used as a sensitizer of CT drugs but also can be used in the initial or maintenance treatment of recurrent tumors (12). Chen et al. found that the patients with liver cancer treated by TACE combined with apatinib, the objective response rate (ORR) was higher than that of TACE alone $(66.7 \%$ and $39.6 \%, \mathrm{P}=0.020$; $45.8 \%$ and $17.6 \%, \mathrm{P}=0.021$ ) (13). Zeng et al. found that low-dose apatinib has significant effect on lung squamous cell carcinoma, the MPFs was 3.1 months, and the disease control rate (DCR) was $46.2 \%$ (14). Lan et al. found that the ORR for apatinib mesylate in combination with etoposide used for the treatment of OC can reach more than $60 \%$ under the condition of controllable toxicity (1). Therefore, it is of great significance to evaluate clinically the safety, and efficacy of apatinib in combination with CT in the treatment of OC.

Evidence-based medicine emphasizes clinical decisions to be made based on the best available evidence on the basis of attaching importance to the clinical experience of doctors and respecting the values of patients (15). Based on highquality clinical randomized controlled trials, systematic review/meta-analysis with rigorous methodology and clear report is the highest level of evidence recognized in the 
Table 1 The search words and strategy of the PubMed database

\begin{tabular}{|c|c|}
\hline No. & Search strategies \\
\hline$\# 1$ & "Apatinib"[Mesh] \\
\hline \#2 & $\begin{array}{l}\text { "rivoceranib mesylate"[Title/Abstract] } \\
\text { OR"YN968D1"[Title/Abstract] OR"Rivoceranib"[Title/ } \\
\text { Abstract] OR"apatinib mesylate"[Title/Abstract] }\end{array}$ \\
\hline$\# 3$ & "Drug Therapies"[Mesh] \\
\hline$\# 4$ & $\begin{array}{l}\text { "chemotherapy"[Title/Abstract] } \\
\text { OR"chemotherapies"[Title/Abstract] } \\
\text { OR"pharmacotherapy"[Title/Abstract] } \\
\text { OR"Pharmacotherapies"[Title/Abstract] }\end{array}$ \\
\hline$\# 5$ & "Ovarian Neoplasm"[Mesh] \\
\hline$\# 6$ & $\begin{array}{l}\text { "ovarian cancer"[Title/Abstract] OR"ovarian } \\
\text { cancers"[Title/Abstract] OR"ovary neoplasm"[Title/ } \\
\text { Abstract] OR"ovary neoplasms"[Title/Abstract] OR"ovary } \\
\text { Cancer"[Title/Abstract] OR"ovary cancers"[Title/Abstract] } \\
\text { OR"cancer of ovary"[Title/Abstract] OR"cancer of the } \\
\text { ovary"[Title/Abstract] }\end{array}$ \\
\hline$\# 7$ & \#10R\#2 \\
\hline \#8 & \#3OR\#4 \\
\hline$\# 9$ & \#5OR\#6 \\
\hline$\# 10$ & \#7AND\#8AND\#9 \\
\hline
\end{tabular}

current health care field (16). In this study, the Cochrane system evaluation method was used to analyze and evaluate the clinical trials of apatinib in combination with CT for the treatment of OC, and to further study and analyze whether it could be a safe and effective treatment for OC, to provide the basis for clinical decision-makers.

We present the following article in accordance with the PRISMA 2020 reporting checklist (available at https:// dx.doi.org/10.21037/apm-21-1662).

\section{Methods}

\section{Search strategy}

Related literature was extensively searched, using search engines such as PubMed, the Web of Science, EMBASE, CNKI, Cochrane Library, CBM, VIP, and the Wanfang database. Clinical studies, related to the use of a combination of apatinib and CT for the treatment of OC, was searched by using the aforementioned databases. The initial search was performed from the establishment of the database to September 2020. At the same time, the references of the studies included were traced to supplement the potential studies that met the inclusion criteria. Keywords used to search the literature were included "Apatinib", "rivoceranibmesylate", "YN968D1", "Rivoceranib", "apatinib mesylate", "Drug Therapies", "chemotherapy", "Chemotherapies", "Pharmacotherapy", "Pharmacotherapies", "ovarian cancer", "Ovarian Neoplasm”, "Ovary Neoplasms”, "Ovary Cancer”, "Cancer of Ovary", "Cancer of the Ovary". No language limits were applied. Free words and subject words are used in the retrieval (Table 1).

\section{Selection criteria}

(I) Many participants were recruited for a clinical comparative study and received either apatinib plus CT or CT alone, regardless of randomization, assignment concealment, or blinding. (II) Participants: the subjects included must be patients diagnosed with OC. (III) Intervention measures: the experimental group was given apatinib based on the treatment criteria designed for the control group, while the control group received only basic CT. The dosage, dosage form, method, and time of the two groups were not limited. (IV) The outcomes consisted of tumor response, adverse reactions (ADRs) due to treatment, and SR. The modified response evaluation criteria in solid tumors (RECIST) criteria, categorized as partial remission $(\mathrm{PR})$, complete remission (CR), progressive disease (PD), and stable disease (SD) was used to evaluate the treatment efficacy against tumor $(17,18)$. DCR is represented by CR + $\mathrm{PR}+\mathrm{SD}$ and the ORR is represented by $\mathrm{CR}+\mathrm{PR}$.

\section{Exclusion criteria}

Exclusion of the studies was carried out if any of the following criteria were met: (I) non-Chinese and NonEnglish literature. (II) Information in their original form could not be achieved upon contacting the author in case the original data were not extracted. (III) The literature was comprising multiple publications, duplicate records, animal experiments, clinical case studies, or similar type studies, (IV) and Literature with inconsistent outcome indicators.

\section{Extraction of data and evaluation of the literature quality}

The literature was searched by two researchers independently. They extracted the data and the results were also crosschecked by using different sources. In the case of disagreement, it was settled down through negotiation or 


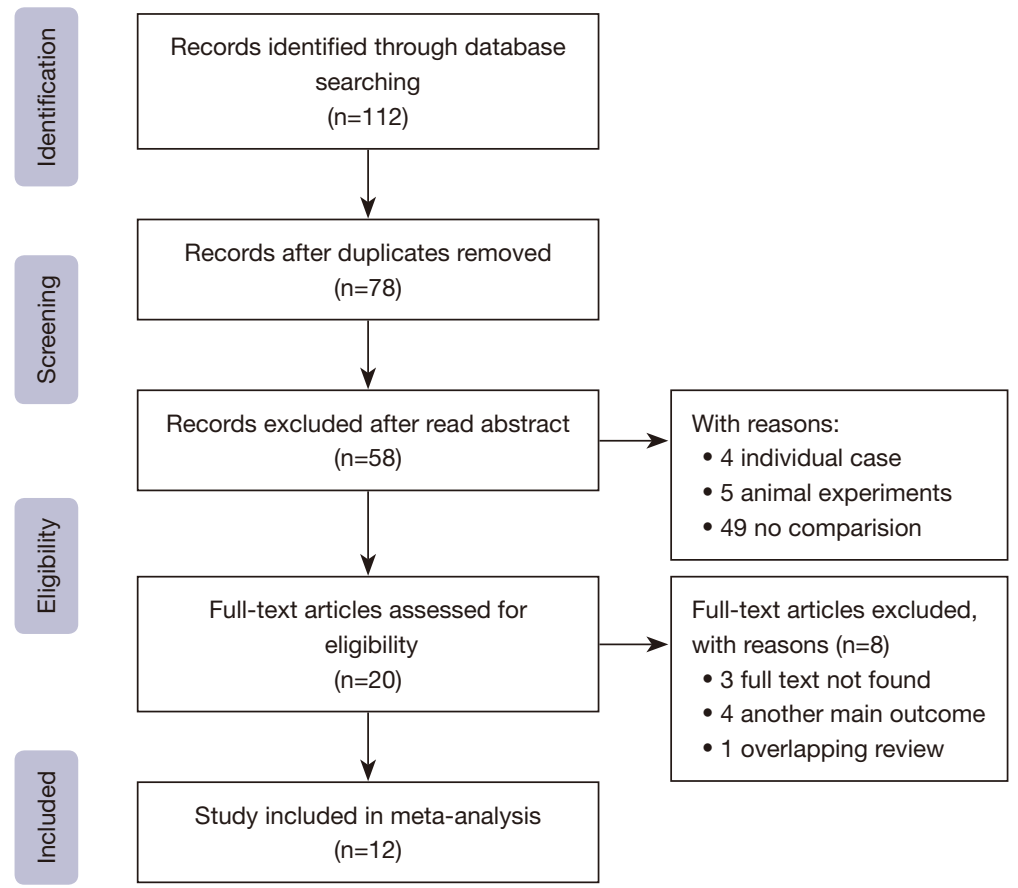

Figure 1 Flow diagram of the selection process.

discussion with a third party. While searching the literature, the title and abstract of a paper or article were read followed by reading the full text of the paper to identify whether the article should be included or not. The criteria for data to be extracted consisted of (I) basic information of the included study such as the title of publication, first author, year in which article was published, etc.; (II) basic characteristics and intervention measures of control and experimental group; (III) key information of bias risk assessment; (IV) outcome indicators. The risk of deviation for each qualified study was evaluated following Cochrane reviewer's manual 5.1.0 (19).

\section{Statistical analysis}

The analyses of the current study were performed using Revman 5.4 software (Cochrane; London, UK). Risk ratio (OR) was used as a statistic in counting the data, weighted mean difference (WMD) was used as a statistic in the data measurement, and $95 \%$ confidence interval (CI) was provided for statistical results. Analysis of the heterogeneity among the included research results was performed using the chi-square test (the test level was $\alpha=0.1$ ), and the size of heterogeneity was quantitatively judged by combining it with $\mathrm{I}^{2}$. If no significant statistical heterogeneity was observed $\left(\mathrm{I}^{2} \leq 50 \%, \mathrm{P} \geq 0.1\right)$, then for merging the results, the fixed-effect model was used. If there is a significant statistical heterogeneity found $\left(\mathrm{I}^{2}>50 \%, \mathrm{P}<0.1\right)$, then for merging the results, the random effect model was used. The sensitivity analysis method was performed to explore the source of heterogeneity. The publication bias test was conducted to identify any asymmetry among studies.

\section{Results}

\section{Search results}

A total of 112 related literatures were obtained from the initial examination, after review the title and authors, 34 literatures were excluded with the reason of duplications. After read the abstracts, 4 individual cases, 5 animal experiments and 49 no comparision studies were excluded. After review the full-text articles, 8 literatures were excluded because 3 full texts were not found, 4 studies have another main outcome and 1 overlapping review. Finally, 12 literatures were finally included after layer-bylayer screening (20-31). All the literatures included 698 patients, including 340 cases in the experimental group and 358 cases in the control group. The specific screening process is shown in Figure 1. 
Table 2 Main characteristics of the 12 studies included in the meta-analysis

\begin{tabular}{|c|c|c|c|c|c|c|c|}
\hline Authors, year & Nation & Line & Age, Exp/con & Therapeutic regimen, Exp/con & $\begin{array}{l}\text { Patients, } \\
\text { Exp/con }\end{array}$ & $\begin{array}{c}\text { Enrollment } \\
\text { period }\end{array}$ & Outcomes \\
\hline Feng et al. (20), 2019 & China & I-IV & $45.80 \pm 5.70 / 46.20 \pm 6.10$ & Apatinib + DDP/DDP & $30 / 30$ & 2017.1-2019.1 & (1)(2) (3) \\
\hline Li et al. (22), 2017 & China & II & $55.00 \pm 14.00 / 52.00 \pm 13.00$ & Apatinib + taxane/taxane & $10 / 20$ & 2016.2-2016.11 & (1)(2) (3) \\
\hline Li (23), 2018 & China & III-IV & $26-70 / 30-74$ & Apatinib + taxane/taxane & $21 / 25$ & 2016.2-2017.11 & (1)(2) (3) \\
\hline Ran et al. (25), 2020 & China & III-IV & $52.10 \pm 12.19 / 51.37 \pm 12.53$ & Apatinib + GEMOX/GEMOX & $41 / 41$ & 2013.2-2017.2 & (1) (2) \\
\hline Shao et al. (26), 2019 & China & No & $64.28 \pm 1.63 / 58.55 \pm 1.45$ & Apatinib + TP/TP & $14 / 14$ & 2018.7-2019.3 & (1) (2) (3) \\
\hline Wang et al. (27), 2019 & China & No & $50.93 \pm 13.72 / 50.42 \pm 13.85$ & Apatinib + DP/DP & $39 / 39$ & 2016.1-2018.1 & (1)(2) (3) \\
\hline Qiu et al. (31), 2020 & China & III-IV & $47.39 \pm 4.87 / 47.39 \pm 4.87$ & Apatinib + paclitaxel/paclitaxel & $45 / 45$ & 2014.7-2016.3 & (1) (2) \\
\hline
\end{tabular}

Outcome: (1) ORR, (2) DCR, (3) adverse events; Con, control group (CT alone group); Exp, experimental group (apatinib targeted therapy plus CT). DDP, docetaxel I + cisplatin chemotherapy; GEMOX, gemcitabine + oxaliplatin; TP, paclitaxel + cisplatin; DP, docetaxel + cisplatin; PH, pirarubicin hydrochloride + paclitaxel liposome; CT, chemotherapy; ORR, objective response rate; DCR, disease control rate.

\section{Patient characteristics}

The trials included in this study were conducted in China. Collectively, 343 patients with an advanced stage of OC received apatinib-cum-CT as a treatment, and around 361 patients received only CT. Detailed information regarding trials and patients included in this study is presented in Table 2.

\section{Quality assessment}

The assessment of the bias risk is shown in Figure 2. Among the included studies, one was found to be having a low risk, and the rest of the 5 were not true randomized controlled trials. None of the included studies provided clear details of the risk detection and performance. The attrition risks of the included trials were found to be low; and due to selective reporting, five trials were found with unclear risk.

\section{Meta-analysis results}

\section{ORR}

In six included $(22,23,25,27,28,31)$ studies involving 364 patients, the meta-analysis showed that the difference was statistically significant (OR $=3.19,95 \%$ CI: 2.06-4.94, $\mathrm{P}<0.00001)$, as shown in Figure 3.

\section{DCR}

In six included $(22,23,25,28,30,31)$ studies involving 308 patients, the meta-analysis showed that the difference was statistically significant (OR $=4.97,95 \%$ CI: $2.90-8.52$, $\mathrm{P}<0.00001)$, as shown in Figure 4.

\section{Therapeutic efficacy assessments}

As presented in Table 3, the results in pooled form revealed that the patients who received a combined therapy showed significantly improved CR, PR, ORR, and DCR (CR: OR $=3.17$, 95\% CI: $1.87-5.38, \mathrm{P}<0.00001$; PR: OR =2.00, 95\% CI: 1.45 c2.75, P<0.00001; ORR: OR $=3.19,95 \%$ CI: 2.06-4.94, P<0.00001; DCR: OR =4.97, 95\% CI: 2.90-8.52, $\mathrm{P}<0.00001)$ and $\mathrm{PD}$ was found to be significantly decreased (OR $=0.29,95 \%$ CI: $0.20-0.43, \mathrm{P}<0.00001$ ), while the SD was observed not significantly different from the group of patients receiving only CT (OR $=0.75,95 \%$ CI: $0.54-1.05$, $\mathrm{P}=0.09$ ). The $\mathrm{OR}$ rate was analyzed by using fixed effect models because of low heterogeneity.

\section{AEs assessment}

This meta-analysis also evaluated the safety of apatinib targeted therapy. As shown in the Table 4, group of patients treated with a combination therapy of apatinib and CT revealed higher rate of proteinuria $(\mathrm{OR}=0.17,95 \% \mathrm{CI}$ : 


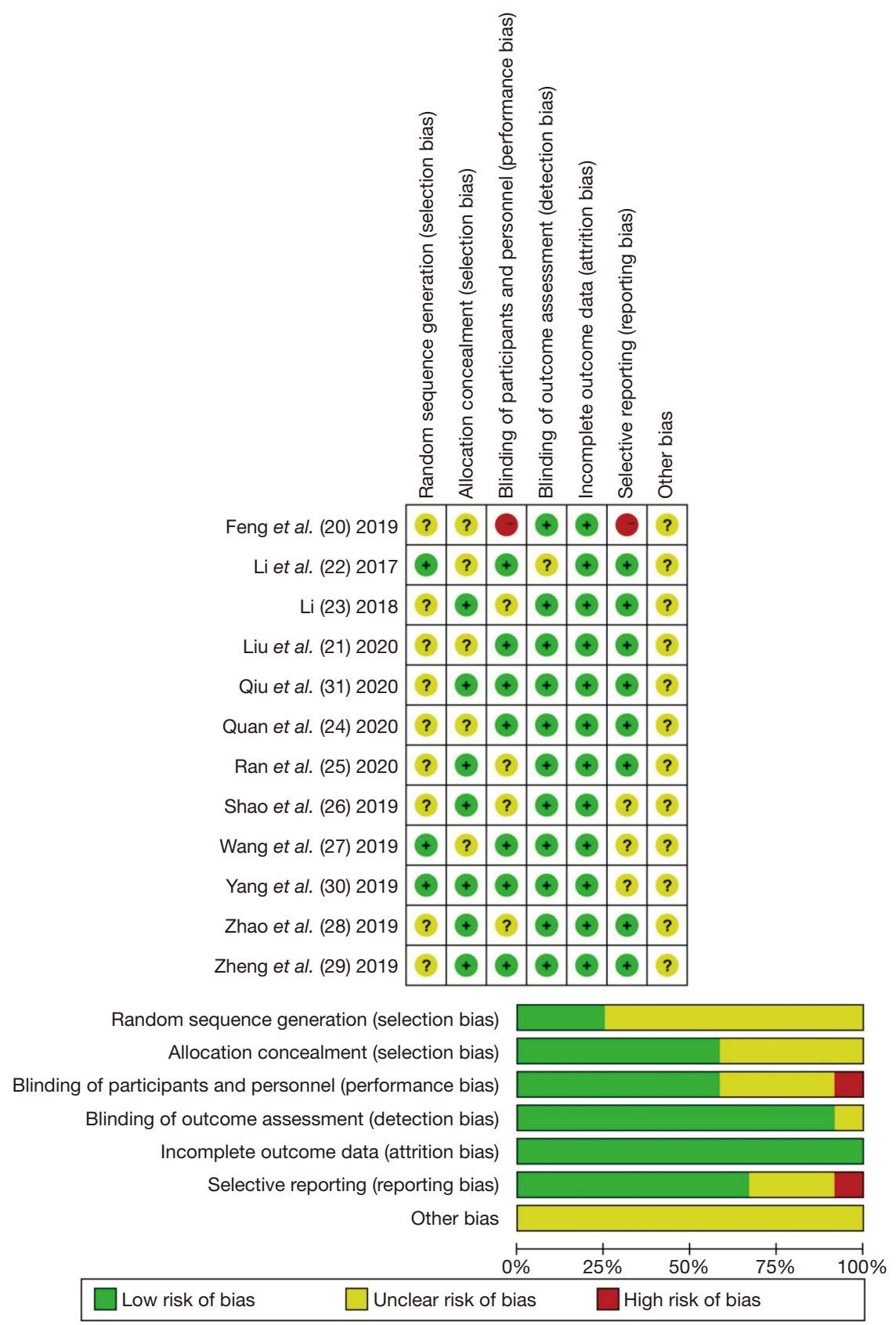

Figure 2 (A) Risk of bias summary: review of the authors' judgments about each risk of bias item for the included studies, (B) risk of bias graph: review of the authors' judgments about each risk of bias item presented as percentages across all included studies. Each color represents a different level of bias: red for high risk, green for low risk, and yellow for unclear risk of bias (20-31).

0.04-0.68, $\mathrm{P}=0.01$ ), whereas analyses of the symptoms such as nausea and vomiting, hand foot syndrome, hypertension, diarrhea, myelosuppression and leucopenia did not reveal any significant differences (Nausea and vomiting: OR $=1.10,95 \% \mathrm{CI}: 0.67-1.79, \mathrm{P}=0.71$; hand foot syndrome:
OR $=1.73,95 \%$ CI: 0.97-3.10, $\mathrm{P}=0.06$; hypertension: $\mathrm{OR}$ $=1.18,95 \%$ CI: $0.73-1.91, \mathrm{P}=0.51$; diarrhea: $\mathrm{OR}=1.05$, 95\% CI: 0.56-1.97, $\mathrm{P}=0.87$; leucopenia: $\mathrm{OR}=1.22,95 \%$ CI: $0.70-2.12, \mathrm{P}=0.48$; myelosuppression: $\mathrm{OR}=0.82,95 \%$ CI: $0.24-2.83, \mathrm{P}=0.62$ ) 


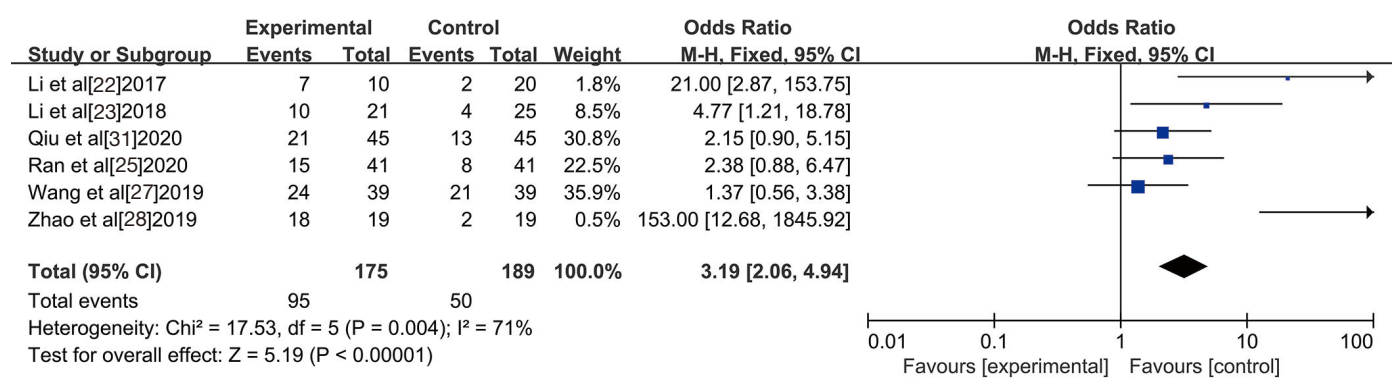

Figure 3 Forest plot of the comparison of the ORR between the experimental and control group $(22,23,25,27,28,31)$. Control group, CT alone group; experimental group, apatinib targeted therapy plus CT. The fixed effects meta-analysis model (Mantel-Haenszel method) was used. CI, confidence interval; CT, chemotherapy; OR, odds ratio; ORR, overall response rate.

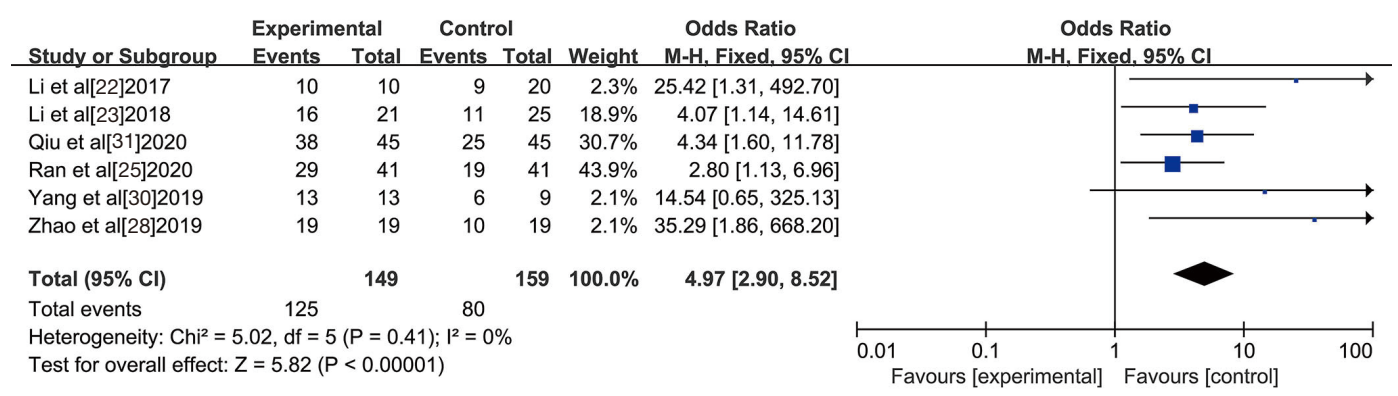

Figure 4 Forest plot of the comparison of the DCR between the experimental and control group $(22,23,25,28,30,31)$. Control group, CT alone group; experimental group, apatinib targeted therapy plus CT. The fixed effects meta-analysis model (Mantel-Haenszel method) was used. CI, confidence interval; CT, chemotherapy; OR, odds ratio; ORR, overall response rate.

Table 3 Comparison of CR, PR, SD, PD, ORR, and DCR between the experimental and control groups

\begin{tabular}{|c|c|c|c|c|c|c|c|c|c|}
\hline Parameter & Studies & \multicolumn{2}{|c|}{ Exp } & \multicolumn{2}{|c|}{ Con } & \multicolumn{2}{|c|}{ Heterogeneity } & \multicolumn{2}{|c|}{ Pooled effect } \\
\hline $\mathrm{CR}$ & 12 & 67 & 344 & 32 & 354 & 0 & 0.65 & $3.17(1.87,5.38)$ & $<0.00001$ \\
\hline PR & 12 & 140 & 344 & 90 & 354 & 59 & 0.005 & $2.00(1.45,2.75)$ & $<0.00001$ \\
\hline SD & 12 & 87 & 344 & 109 & 354 & 41 & 0.07 & $0.75(0.54,1.05)$ & 0.09 \\
\hline ORR & 6 & 95 & 175 & 50 & 189 & 72 & 0.004 & $3.19(2.06,4.94)$ & $<0.00001$ \\
\hline DCR & 6 & 125 & 149 & 80 & 159 & 0 & 0.41 & $4.97(2.90,8.52)$ & $<0.00001$ \\
\hline
\end{tabular}

Con, control group (CT alone group); Exp, experimental group (apatinib targeted therapy plus CT). CR, complete response rates; CT, chemotherapy; PR, partial response rates; SD, stable disease rates. PD, progressive disease rates; ORR, overall response rate; DCR, disease control rate; OR, odds ratio; $\mathrm{Cl}$, confidence intervals. 
Table 4 Comparison of AEs between the experimental and control groups

\begin{tabular}{|c|c|c|c|c|c|c|c|c|c|}
\hline Outcome & Studies & \multicolumn{2}{|c|}{ Exp } & \multicolumn{2}{|c|}{ Con } & \multicolumn{2}{|c|}{ Heterogeneity } & \multicolumn{2}{|c|}{ Pooled effect } \\
\hline Nausea and vomiting & 10 & 87 & 248 & 98 & 262 & 0 & 0.83 & $1.10(0.67,1.79)$ & 0.71 \\
\hline Hand foot syndrome & 7 & 73 & 187 & 83 & 197 & 66 & 0.02 & $1.73(0.97,3.10)$ & 0.06 \\
\hline Hypertension & 10 & 96 & 286 & 103 & 296 & 0 & 0.49 & $1.18(0.73,1.91)$ & 0.51 \\
\hline Leukopenia & 8 & 76 & 196 & 84 & 206 & 0 & 0.93 & $1.22(0.70,2.12)$ & 0.48 \\
\hline Myelosuppression & 2 & 5 & 79 & 6 & 79 & 0 & 0.75 & $0.82(0.24,2.83)$ & 0.62 \\
\hline Proteinuria & 4 & 23 & 105 & 37 & 109 & 0 & 0.11 & $0.17(0.04,0.68)$ & 0.01 \\
\hline
\end{tabular}

Con, control group (CT alone group); Exp, experimental group (apatinib targeted therapy plus CT). OR, odds ratio; Cl, confidence intervals.

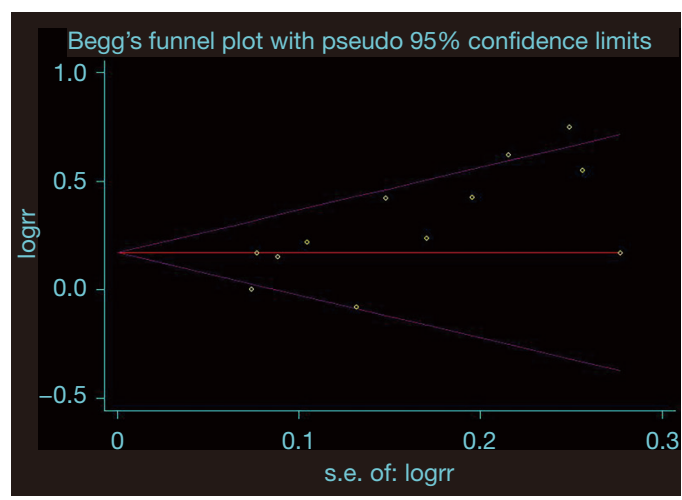

Figure 5 Funnel plot of percentage of ORR. ORR, overall response rate.

\section{Publication bias}

drawn for the studies on outcome of ORR, but without complete symmetry, as presented in Figure 5.

\section{Sensitivity analysis}

We conducted a sensitivity analysis to explore the sources of heterogeneity in treatment regimens, apatinib dose, sample size, and related study types in ORR. As shown in Figure 6, our analysis showed that no significant differences were found between different treatment regimens, apatinib doses, sample sizes, and study types.

\section{Discussion}

The clinical symptoms of early OC are difficult to be detected, thus leading to its high rate of mortality rate. In most cases, cancer has progressed to an advanced stage at

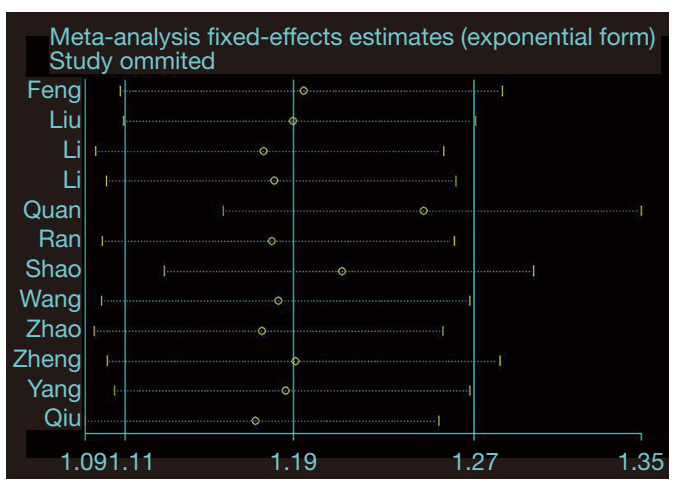

Figure 6 Sensitivity analysis of ORR. ORR, overall response rate.

the time of its diagnosis. In the past 20 years, paclitaxel combined with platinum has been the global first-line CT program for OC patients (32). However, when cytotoxic drugs kill cancer cells, they also mediate adverse events to the body. As a result, CT is either stopped or its dose is reduced, thus affecting the efficacy of surgery and the patients' quality of life (33). Therefore, there is an urgent need to develop new targeted therapeutic drugs for the effective treatment of OC $(34,35)$. In recent times, with the progress in the molecular biology of tumor and epigenetics, more and more drugs such as erlotinib, gefitinib, and apatinib against different targets are being used to treat patients with malignant tumors (36-38). Therefore, apatinib combined with conventional CT may be a new option to inhibit the growth of OC, especially for patients who have received multiple CT routes or are resistant to CT.

Basic research has confirmed that tumor neovascularization is a major factor involved in the occurrence and process of 
the development of malignancy of tumors (39). Abnormal activation of the VEGF/VEGFR-2 signaling pathway is the key factor of neovascularization $(40,41)$. In a variety of malignant tumors, its abnormal activation can promote the vascular endothelial cells proliferation and migration, inhibit the endothelial cells apoptosis and reorganization, thus it is closely related to the degree of malignancy and prognosis of the tumor (42). Apatinib is a new VEGFR-2 targeted blocker developed in China. It blocks VEGF/VEGFR-2 signal pathway with high selectivity and strong effect, thus inhibits the activation of mitogen-activated protein kinases (MAPKs), and hinders the vascular endothelial cells proliferation and migration. This in turn leads to the inhibition of tumor neovascularization and killing of cancer cells (43). At present, apatinib has been reported to treat the OC. Li Jing's (44) study showed that apatinib can effectively down-regulate the expression of CDK2 and p21 in A2780 cells, inhibit the cell proliferation in OC, and increase the sensitivity of OC towards paclitaxel. Despite the statistical analysis of many earlier published clinical trials, the exact therapeutic effects and safety have not been systematically determined so far, owing to the sample size variability in these clinical trials. In addition, in different clinical trials, different application schemes may lead to diverse therapeutic effects. In this study, an extensive online search was conducted followed by a rigorous comparison and combination for classification in order to present a clear, and systematic conclusion.

Results of the current meta-analysis show that apatinib targeted therapy in combination with CT is more effective than CT alone. Compared with the CT group, PR, PD, CR, ORR, and DCR were found to be significantly higher $(\mathrm{P}<0.05)$ for the group of combined treatment. The main adverse effects observed were nausea, pain in hand and foot, hypertension, leukopenia, and many more. These ADRs were from mild to a moderate level in terms of rampancy and can be overcome by appropriate treatment, including a temporary reduction in dose or other symptomatic treatment. It is worth mentioning that these studies did not report any disability or death related to treatment. Therefore, the combination of apatinib along with CT did not increase the ADRs, which means that the combination therapy can improve the efficacy of OC without increasing ADRs. These findings suggest that opioid targeted therapy improves the efficacy of CT through tumor angiogenesis inhibition, which in turn improves the quality of a patient's life.

However, some limitations also exist in our research. First of all, most of the studies were conducted for patients with OC in China. Probably because apatinib is an independently produced drug in China, mainly used in these areas. Secondly, there are only twelve articles available about the combination of apatinib and CT. This may be because combination therapy is a new treatment strategy, and further studies are currently being carried out, which is confirmed in literature search. We will continue to focus on the results and outcomes of subsequent studies and will update the current metaanalysis on time. Third, it is difficult to analyze the survival rate because the survival time in each study is inconsistent. Furthermore, the medication method and treatment course of apatinib, follow-up duration, and evaluation of adverse events after treatment were not completely consistent and reliable.

\section{Conclusions}

In summary, our study confirmed that apatinib in combination with CT for the treatment of patients with advanced OC is an effective treatment for advanced OC patients. Apatinib in combination with CT markedly enhances the ORR and DCR for advanced OC. However, this combined treatment could lead to greater rates of proteinuria, hypertension, albuminuria, and hand-foot syndrome and so on. Therefore, the benefits and risks should be considered before treatment.

\section{Acknowledgments}

Funding: This study was supported by the ScienceTechnology Foundation for the health care industry of Gansu Province of China (Grant NO: GSWSKY2020-77) and Key Discipline project Fund of Gansu Provincial Hospital of China (Grant NO: 18GSSY1-7) and Science-Technology Foundation for the health care industry of Gansu Province of China (Grant NO: GSWSKY2020-17).

\section{Footnote}

Reporting Checklist: The authors have completed the PRISMA 2020 reporting checklist. Available at https:// dx.doi.org/10.21037/apm-21-1662

Conflicts of Interest: All authors have completed the ICMJE uniform disclosure form (available at https://dx.doi. org/10.21037/apm-21-1662). The authors have no conflicts 
of interest to declare.

Ethical Statement: The authors are accountable for all aspects of the work in ensuring that questions related to the accuracy or integrity of any part of the work are appropriately investigated and resolved.

Open Access Statement: This is an Open Access article distributed in accordance with the Creative Commons Attribution-NonCommercial-NoDerivs 4.0 International License (CC BY-NC-ND 4.0), which permits the noncommercial replication and distribution of the article with the strict proviso that no changes or edits are made and the original work is properly cited (including links to both the formal publication through the relevant DOI and the license). See: https://creativecommons.org/licenses/by-ncnd $/ 4.0 /$.

\section{References}

1. Lan CY, Wang Y, Xiong Y, et al. Apatinib combined with oral etoposide in patients with platinum-resistant or platinum-refractory ovarian cancer (AEROC): a phase 2, single-arm, prospective study. Lancet Oncol 2018;19:1239-46.

2. Jin M, Cai J, Wang X, et al. Successful maintenance therapy with apatinib inplatinum-resistant advanced ovarian cancer and literature review. Cancer Biol Ther 2018;19:1088-92.

3. Bray F, Ferlay J, Soerjomataram I, et al. Global cancer statistics 2018: GLOBOCAN estimates of incidence and mortality worldwide for 36 cancers in 185 countries. CA Cancer J Clin 2018;68:394-424.

4. Boussios S, Moschetta M, Zarkavelis G, et al. Ovarian sex-cord stromal tumours and small cell tumours: Pathological, genetic and management aspects. Crit Rev Oncol Hematol 2017;120:43-51.

5. Han YF, Zhang YM, Li FM, et al. The short-term effect and clinical observation of apatinib mesylate combined with pemetrexed and lobaplatin in the treatment of recurrent ovarian cancer. Chinese Journal Of Family Planning \& Gynecotokology 2020;12:76-80.

6. Moschetta M, Boussios S, Rassy E, et al. Neoadjuvant treatment for newly diagnosed advanced ovarian cancer: where do we stand and where are we going? Ann Transl Med 2020;8:1710.
7. Liang XY, Huang LS, Hu YX, et al. Efficacy of the Huangqi Fuzheng decoction on immune function in patients with ovarian cancer after chemotherapy. Clinical Journal of Chinese Medicine 2017;9:142-4.

8. Bookman MA. Trials with impact on clinical management: first line. Int J Gynecol Cancer 2009;19 Suppl 2:S55-62.

9. Korkmaz T, Seber S, Basaran G. Review of the current role of targeted therapies as maintenance therapies in first and second line treatment of epithelial ovarian cancer; In the light of completed trials. Crit Rev Oncol Hematol 2016;98:180-8.

10. Boussios S, Karihtala P, Moschetta M, et al. Veliparib in ovarian cancer: a new synthetically lethal therapeutic approach. Invest New Drugs 2020;38:181-93.

11. Zhang H. Apatinib for molecular targeted therapy in tumor. Drug Des Devel Ther 2015;9:6075-81.

12. Li J, Zhao X, Chen L, et al. Safety and pharmacokinetics of novel selective vascular endothelial growth factor receptor-2 inhibitor YN968D1 in patients with advanced malignancies. BMC Cancer 2010;10:529.

13. Chen S, Yu W, Zhang K, et al. Comparison of the efficacy and safety of Transarterial chemoembolization with and without Apatinib for the treatment of BCLC stage C hepatocellular carcinoma. BMC Cancer 2018;18:1131.

14. Zeng DX, Lei W, Wang CG, et al. Low dosage of apatinib monotherapy as rescue treatment in advanced lung squamous cell carcinoma. Cancer Chemother Pharmacol 2019;83:439-42.

15. Djulbegovic B, Guyatt GH. Progress in evidence-based medicine: a quarter century on. Lancet 2017;390:415-23.

16. Ge L, Tian JH, Li YN, et al. Association between prospective registration and overall reporting and methodological quality of systematic reviews: a metaepidemiological study. J Clin Epidemiol 2018;93:45-55.

17. Lencioni R. New data supporting modified RECIST (mRECIST) for Hepatocellular Carcinoma. Clin Cancer Res 2013;19:1312-4.

18. Lencioni R, Llovet JM. Modified RECIST (mRECIST) assessment for hepatocellular carcinoma. Semin Liver Dis 2010;30:52-60.

19. Higgins J, Green S. Cochrane Handbook for Systematic Reviews of Interventions Version 5.1.0. The Cochrane Collaboration; 2011.

20. Feng Y, Wan YY, Wang XW, et al. Clinical effect of 
apatinib combined with chemotherapy on patients with drug-resistant ovarian cancer. Clinical Research and Practice 2019;4:20-1.

21. Liu HM, Cui DM, Li YY. Efficacy of apatinib combined with chemotherapy in the treatment of advanced ovarian cancer after the second-line treatment has failed. Chinese Journal of Clinical Oncology and Rehabilitation 2020;27:316-8.

22. Li Q, Zhao JF, Shi XY, et al. Clinical observation of apatinib combined with chemotherapy for second-line treatment failure in advanced ovarian cancer. Journal of Hebei Medical University 2017;38:1384-7.

23. Li Q. Clinical observation of apatinib combined with chemotherapy for second-line treatment failure in advanced ovarian cancer. Hebei North Hospital, 2018. Available online: https://d.wanfangdata.com.cn/thesis/Ch JUaGVzaXNOZXdTMjAyMTA1MTkSCFkzNDg4MD EzGggzdWkyZDV3Zg\%253D\%253D

24. Quan Y. Effect of Quanying and apatinib combined with chemotherapy in the treatment of drug-resistant ovarian cancer. China Practical Medical 2020;15:117-8.

25. Ran X, Liu XJ. Evaluation of the efficacy and safety of Apatinib combined with GEMOX regimen for advanced refractory ovarian cancer. Chinese Journal of Human Sexuality 2020;29:44-8.

26. Shao RX, Han LL, Chen XH, et al. Clinical efficacy and safety analysis of apatinib combined with TP Regimen in the treatment of recurrent ovarian cancer after platinum chemotherapy. Journal of Practical Gynecologic Endocrinology 2019;6:124-5.

27. Wang XY, Qu F. Clinical study on apatinib combined with DP regime in treatment of advanced ovarian cancer. Drugs \& Clinic 2019;34:2132-6.

28. Zhao LF, Wu CH, Hou DH. The clinical analysis of apatinib combined with chemotherapy for advanced ovarian cancer after failure of second-line treatment. Journal of Clinical Medical Literature (ElectronicEdition) 2019;6:171-4.

29. Zheng CH, Tang ZY, Liu T. Discussion on efficacy of apatinib combined with chemotherapy in the treatment of recurrence of epithelial ovarian cancer. Chinese Journal of Modern Drug Application 2019;13:106-8.

30. Yang M, Liu X, Zhang C, et al. A Study Of Efficacy And Safety With Apatinib Or Apatinib Combined With Chemotherapy In Recurrent/advanced Ovarian Cancer Patients. Cancer Manag Res 2019;11:8869-76.
31. Qiu M, Wu J, Ye X, et al. Clinical efficacy and prognosis of chemotherapy regimen of apatinib combined with paclitaxel in the treatment of advanced ovarian cancer. Int J Clin Exp Med 2020;13:191-9.

32. Ren FF, Zhao TQ. Effect of proteasome inhibitor Oprozomib on ovarian cancer cells to cis-platinum. Medical Journal of Chinese People's Liberation Army 2020;45:405-10.

33. Kampan NC, Madondo MT, McNally OM, et al. Paclitaxel and Its Evolving Role in the Management of Ovarian Cancer. Biomed Res Int 2015;2015:413076.

34. Liu J, Matulonis UA. New strategies in ovarian cancer: translating the molecular complexity of ovarian cancer into treatment advances. Clin Cancer Res 2014;20:5150-6.

35. Webb PM, Jordan SJ. Epidemiology of epithelial ovarian cancer. Best Pract Res Clin Obstet Gynaecol 2017;41:3-14.

36. Han B, Jin B, Chu T, et al. Combination of chemotherapy and gefitinib as first-line treatment for patients with advanced lung adenocarcinoma and sensitive EGFR mutations: A randomized controlled trial. Int J Cancer 2017;141:1249-56.

37. Fountzilas C, Chhatrala R, Khushalani N, et al. A phase II trial of erlotinib monotherapy in advanced pancreatic cancer as a first- or second-line agent. Cancer Chemother Pharmacol 2017;80:497-505.

38. Li J, Qin S, Xu J, et al. Randomized, Double-Blind, Placebo-Controlled Phase III Trial of Apatinib in Patients With Chemotherapy-Refractory Advanced or Metastatic Adenocarcinoma of the Stomach or Gastroesophageal Junction. J Clin Oncol 2016;34:1448-54.

39. Li J, Qin S, Xu J, et al. Apatinib for chemotherapyrefractory advanced metastatic gastric cancer: results from a randomized, placebo-controlled, parallel-arm, phase II trial. J Clin Oncol 2013;31:3219-25.

40. Zhao J, Zhang X, Gong C, et al. Targeted therapy with apatinib in a patient with relapsed small cell lung cancer: A case report and literature review. Medicine (Baltimore) 2017;96:e9259.

41. Kerbel RS. Tumor angiogenesis. N Engl J Med 2008;358:2039-49.

42. Pujade-Lauraine E, Selle F, Weber B, et al. Volasertib Versus Chemotherapy in Platinum-Resistant or -Refractory Ovarian Cancer: A Randomized Phase II Groupe des Investigateurs Nationaux pour l'Etude des Cancers de l'Ovaire Study. J Clin Oncol 2016;34:706-13. 43. Qi R, Wang Y, Bruno PM, et al. Nanoparticle conjugates 
of a highly potent toxin enhance safety and circumvent platinum resistance in ovarian cancer. Nat Commun 2017;8:2166.

44. Li J, Liu QL, Chen L, et al. Apatinib suppresses the

Cite this article as: Qi J, Liu E, Yue H, Chen G, Liu Y, Chen J. Evaluation of safety and efficacy of apatinib combination with chemotherapy for ovarian cancer treatment: a systematic review and meta-analysis. Ann Palliat Med 2021;10(9):9902-9913. doi: 10.21037/apm-21-1662 proliferation of A2780 cells and increases cellular sensitivity to pacli-taxel. Progress in Obstetrics and Gynecology, 2018;27:46-8. 